\title{
Critical Analysis of the Healthcare Response to Burn-Pit-Related Illnesses for Post-9/11 Iraq and Afghanistan Veterans
}

\author{
Peter J. Lim*, Ali R. Tayyeb* \\ Patricia A. Chin School of Nursing, California State University, Los Angeles, CA, USA \\ Email: *peter.limx5@gmail.com, *atayyeb2@calstatela.edu
}

How to cite this paper: Lim, P.J. and Tayyeb, A.R. (2019) Critical Analysis of the Healthcare Response to Burn-Pit-Related Illnesses for Post-9/11 Iraq and Afghanistan Veterans. Open Journal of Emergency Medicine, 7, 17-27.

https://doi.org/10.4236/ojem.2019.72003

Received: March 19, 2019

Accepted: May 26, 2019

Published: May 29, 2019

Copyright () 2019 by author(s) and Scientific Research Publishing Inc. This work is licensed under the Creative Commons Attribution International License (CC BY 4.0).

http://creativecommons.org/licenses/by/4.0/

\begin{abstract}
A serious concern among post-9/11 Iraq and Afghanistan veterans (IAV) are health conditions from military and environmental exposure while serving. Many veterans are becoming suddenly ill and experiencing symptoms that have been associated with exposure to burning trash or "burn pits" set up to destroy waste produced by the military. IAVs are exhibiting respiratory conditions and other numerous health issues from exposure to burn pits during deployments. From the US military operations to the Middle East, $70 \%$ of the military personnel reported respiratory illnesses, with $17 \%$ experiencing symptoms serious enough to require medical care. Despite growing concerns, little research has been conducted on the health conditions of returning veterans. Many veterans have difficulty gaining appropriate access to care, unable to travel long distances to Veterans health facility, or have to deal with long wait times to see a provider. As a result, many veterans utilize civilian emergency rooms and healthcare facilities. Most healthcare facilities are not familiar with military-related health conditions. To address and prevent delays in care, it is helpful to have a veteran-specific screener questionnaire at the point of healthcare access. Getting a better understanding of the background and history of veterans can aid in accurately triaging this community and lead to better health care outcomes. Collecting and maintaining data regarding health issues that can arise from burn pit exposures can help direct the care of this specific community of veterans. In seeking to improve the understanding of IAV health issues from exposure to burn pits, the aim of this article is to help educate and raise awareness, and assist health care professionals to better triage and direct the care or resources necessary to help this underserved and at-risk community of our veterans.
\end{abstract}

\section{Keywords}

Veterans, Burn Pit, Screener 


\section{Introduction}

In the US, nearly 3 million service members have been deployed to war zones since 2001 [1]. Past military conflicts have led to a rise in infections due to the accumulation and management of trash. To address the issue of trash, the military started open-air burning pits that became the primary solid waste management in Iraq and Afghanistan. In 2009, the US restricted the usage of burn pits in Iraq, but the use of burn pits continued in Afghanistan [2]. The Department of Defense (DoD) estimates that such burn sites destroy about 60,000 - 85,000 pounds of solid waste per day [3]. A recent survey reports that about $80 \%$ of IAVs were exposed to burn pits during their deployments, and over $60 \%$ reported having symptoms such as shortness of breath, increased coughing and mucus build up, joint and abdomen pain, fatigue and malaise [4]. Health problems associated with burn pits must be understood in order to assess and screen veterans for care. Veterans suffer from a variety of conditions and encounter various types of health issues, including post-traumatic stress disorder (PTSD), traumatic brain injury (TBI), depression, multiple forms of musculoskeletal issues, poly-trauma, hazardous material exposures, chronic pain, substance abuse disorder, and suicide ideation, among others. These health conditions are further exacerbated by non-health factors, such as socio-economic issues, financial issues, unemployment, access to care, substance abuse, and homelessness [5]. There are many factors that can complicate veterans' health situations and prevent them from receiving the care they need. In an attempt to address one facet of this problem, the Veterans Affairs (VA) administration has sought to better understand burn pit exposure. Registry websites, such as Burn Pits 360 and, later, the Veteran Affairs Burn Pit Registries, have been established to document and track information regarding burn pit exposure. These registries help in obtaining data for screening but lack depth in gathering data and connecting with the affected groups. Additionally, asking the appropriate questions, following up on reported health issues and assisting in health care plan can help researchers' trend and anticipate the type and level of care needed for specific health issues.

\section{Variables That Contribute to Respiratory Issues}

Many contributing factors related to military service impact the health and quality of life of service members and veterans. Among the concerned issues, one is that of respiratory health. An emerging contributing factor for IAVs respiratory health is burn pit exposure, which contributes to many health conditions, especially respiratory disorders. For this reason, a thorough and concise survey or screening process is needed. Many other variables also contribute to respiratory issues among IAVs. For example, some IAVs have had jobs that exposed them to inhalants, such as isocyanate-containing paints used on vehicles and welding fumes when fixing vehicles. JP-8 jet fuel fumes are also a work-place hazard that can cause health problems similar to those caused by burn pits. Other environmental pollutants to be considered include desert dust particulate matter, diesel 
exhaust, cigarette smoke, aluminum, cadmium and lead. Iraqi dust has been known to cause increased incidents of lung inflammation and fibrosis as a result of the increased regulation of luekin 2 and decreased regulatory T cells. Many IAVs have been exposed to the environmental debris through dust storms. Smoking is also a major respiratory health concern for many IAVs. According to a study conducted by the Millennium Cohort, service members that were deployed to a combat theater had higher incidence of smoking and greater difficulty trying to quit smoking [6]. In addition, many IAVs have been exposed to improvised explosive devices (IEDs). The fumes of an IED explosion could cause metal particulates to be inhaled and bonded to the iron in IAVs' lungs [7]. IAVs hit with IEDs may have TBIs that can lead to pulmonary injury from barotrauma. TBI affects multiple organ systems and can affect the respiratory system with acute lung injury or blast lung injury. Research regarding long-term effects of TBI for respiratory issues represents a gap in knowledge that should be addressed in future research [6].

\section{Health Problems Associated with Burn Pits}

As noted above, the biggest health concern related to burn pit exposure is that of respiratory issues. Abnormal incidences of health issues have been associated with IAVs who have been exposed to burn pit fumes. According to Falvo et al. (2016), 75\% of the post-9/11 veterans surveyed had normal lung volumes and spirometry on pulmonary function testing; however, an isolated reduction in lung diffusing capacity (DLCO) was observed in 30\% of the sample. An isolated reduction in DLCO is a rare pattern in primary-care-seeking dyspneic patients but is commonly associated with underlying pulmonary disease. Post-9/11 veterans with respiratory complaints and an isolated reduction in DLCO should undergo further evaluation [8]. New onset of shortness of breath and cough was 1.7 times elevated in service members that deployed not related to the issues with smoking [9]. Asthma related to allergy is the fourth most frequent diagnosis of IAVs and is a common problem related to burn pit exposures. Vocal cord dysfunction (VCD) can be suspected in IAVs who have not responded to asthma treatment. When an abnormal flow-volume loop with truncation of the inspiratory limb is found while conducting a pulmonary function test (PFT), a direct laryngoscopy can help in diagnosing VCD [7]. Another study found a consistent and significant pattern of prevalence of chronic obstructive pulmonary disease (COPD) and asthma in IAVs between 2003 to 2011 [6]. In self-reported burn pit smoke exposure, the majority of the health problems correlated with self-reported occurrence of emphysema, chronic bronchitis, COPD and hypertension, although no documentation of significant dose-response association was found [10]. Studies of the VA registry findings suggest that asthma and chronic bronchitis were associated with dust storm exposure than open burn pits; however, it should be noted that these data are less reliable due to the nature of the voluntary registry [11]. Constrictive bronchiolitis has also been found in IAVs exposed to burn pits according to a study conducted on 80 soldiers from Fort Campbell, Kentucky. 
Using a diagnostic biopsy, the research confirmed the diagnosis of constrictive bronchiolitis on soldiers that had served in Iraq and Afghanistan and had inhalational exposure [12]. Constrictive bronchiolitis is difficult to diagnose in healthy patients, and invasive surgical lung biopsy was required for the diagnosis for many of the prior service members. Further studies have diagnosed constrictive bronchiolitis in veterans by using a cryoprobe that provides larger tissue samples and results in better outcomes [13]. Although much of the existing research on burn pits has focused on respiratory issues, further research is needed to diagnose other health problems related to burn pit exposures. Gastrointestinal issues have been found with Eosinophilic esophagitis (EoE), which is usually caused by aeroallergens. Some IAVs have been found to have EoE because of their exposure to burn pits where metal, rubber, solvents, munitions, plastic, painted wood, and other harmful waste products were incinerated. There is a growing interest in understanding the pathophysiology of EoE on the IAV population [14]. Moreover, autoimmune diseases such as lupus and rheumatoid arthritis have also been correlated with burn pit exposure [14]. A significant increase of lupus diagnoses among service members has been associated with personnel deployed to Joint Base Balad in Iraq. IAVs also complain of Chronic Multisystem Illness (CMI), which is classified as having one of the following symptoms: general fatigue, mood and cognitive problems, and musculoskeletal discomfort. Research has shown a slight increase in the risk of CMI from more than 210-day exposure to burn pits [3]. With increasingly high rates of respiratory symptoms and new asthma issues reported in the IAV population, there was a need to better track these problems; as a result, burn pit registries were created. Researchers at Augusta University in Georgia worked on a new study called "Deployed Veterans Have Higher Proportionate Cancer Mortality from Exposure to Open-Air Burn Pits" and discovered that more exposure to burn pits was correlated with higher risk of developing cancers and other ailments [15].

\section{Comparisons between Burn Pits and Trash Fires}

Equating burn pit exposure to other burning events can aid in understanding this type of exposure. The National Center for Atmospheric Research estimates that more than 41 percent of the world's garbage is burned in unregulated open trash fires. Moreover, 29 percent of human-related global emissions of small particulates, 10 percent of mercury and 40 percent of a group of gases known as polycyclic aromatic hydrocarbons (PAHs) come from open trash fires. These pollutants have been known to cause people within the vicinity to suffer from decreased lung function, neurological disorders, cancer, and heart attacks [16]. In Beirut and Mount Lebanon in 2015, a waste management crisis resulted in trash burning in open fires. According to the Human Rights Watch, the people living near the trash fires reported many health problems, including chronic obstructive pulmonary disease, coughing, throat irritation, skin conditions, and asthma [17]. Symptoms similar to these were seen in IAVs who inhaled fumes 
from burn pits that used mainly JP-8 jet fuel to burn trash and debris. Moreover, symptoms were also seen in firefighters and rescue personnel involved in the 9/11 Twin Towers rescue operations. Inhaled fumes from burning jet fuel resulted in rescue personnel experiencing an accelerated decline in lung function and hyper-responsiveness in a methacholine challenge, an indicator of worsening asthma [7]. Children living close to Ground Zero showed a reduction of peak expiratory flow rates and had to be treated repeatedly for respiratory issues associated with asthma [7]. Given the existing evidence associated with trash burning and chemical burns, we can conclude that there is a strong relation between respiratory difficulty and burn pit exposure in Iraq and Afghanistan.

\section{Burn Pit Registries}

Burn pit registries have been created to track health symptoms of burn pit exposure. One of these registries is BurnPits360.org, a voluntary nonprofit civilian website that tracks health symptoms of service members after their deployment to the Middle East. The awareness raised by this registry led to the Open Burn Pits Registry Act, resulting in the VA's creation of the Airborne Hazard and Open Burn Pit (AH\&OBP) registry [7]. The Burn Pits 360 registry does not require any special login, and all the questions are displayed on its first page. The questionnaire collects information such as the following: date and location of deployment; how far the respondent lived and worked from burn pits; status of the respondent's pre-deployment health exam; whether the respondent has had a lung biopsy; whether the respondent has been on oxygen and, if so, how much was required; when the respondent's symptoms started, if any; whether the respondent is a smoker; VA disability compensation, if any; and various symptoms and types of cancer, if any. Additionally, the registry includes a section for the cause of death if the questionnaire is being filled out on behalf of a deceased veteran or service member [18]. According to the VA's burn pit registry fact sheet, the AH\&OBP registry includes not only IAVs but military service members deployed to contingency operations in the Southwest Asia theater after August 2, 1990, or Afghanistan or Djibouti on or after September 11, 2001. The AH\&OBP registry covers more countries and geographical regions than Burn Pits 360, including: Iraq, Afghanistan, Kuwait, Saudi Arabia, Bahrain, Djibouti, Gulf of Aden, Gulf of Oman, Oman, Qatar, United Arab Emirates, Persian Gulf, Arabian Sea, and Red Sea. The AH\&OBP is also a more complex registry to use, requiring the user to login and having a less user-friendly questionnaire and less reliable website performance. However, inconsistencies in both the Burn Pits 360 and the AH\&OBP may significantly impact the reliability of the data being collected. The information gathered from the burn pit registries can aid in the provider's understanding and care of the health symptoms of IAVs. As the research gap related to burn pit exposure makes it difficult for providers to understand the correlation between burn pit exposure and IAV health problems, the burn pit registries can help providers to obtain reliable data for treatment plans. Discharge instructions including information on burn pit registries and how to use 
them can aid the IAV in engaging and actively participating in understanding the disease process and can aid the healthcare community in the research and treatment of burn-pit-related health disorders.

\section{Problems with the Registries}

Unfortunately, many important questions are missing within both the Burn Pits 360 and AH\&OBP registries that could help aid in the diagnosis of multiple health issues related to burn pits. The missing in both registries are questions related to impulse oscillometry, allergy skin testing, contact dermatitis from metals and chemicals, exhaled breath condensate nitric oxide level for eosinophilic airway inflammation, sinusitis, gastroesophageal reflux disease, changes in spirometry, exposure to grinding or metal particulates, and pre-deployment history of allergies or asthma [7]. Burn Pits 360 also allows respondents to report experiences at small operating bases called forward operating bases (FOB) that had burn pits, whereas AH\&OBP only acknowledges established bases. Burn Pits 360 includes sections for death and types of cancer. Reports have been made regarding flaws related to the voluntary and self-report method of the AH\&OBP registry because of their selection of participation, faulty recall, and exaggeration and underestimation of health problems. The questions are thought to be poorly written and its direction to find the main health-related problem. Moreover, about $40 \%$ of participants did not complete the survey, most likely due to its extensive length [19]. Although the registries help in gathering data, many IAVs are not participating in them, either fully or at all. Furthermore, due to both of the registry's problems, it does a poor job of obtaining the details needed for proper data collection. The registry also does not help in understanding the plan of care and history of the patient when they present to facilities and emergency rooms that do not screen for burn pit predisposing factors.

\section{Recommendations}

When veterans come into the healthcare system through a port of entry such as an emergency room, it is important to obtain a reliable history and physical from them. An incomplete medical history of an IAV can result in misdiagnosis and delays in care. When an acute health concern arises or the appointments are not available from a primary provider, an IAV might come to an emergency room. Thus, having a standardized screener questionnaire available at such locations can be helpful in quickly assessing the history of the IAV population and can assist in data collection regarding burn pit exposures for future research. Combining questions from the two registries and adding pertinent questions should aid in diagnosis and future care.

\section{Emergency Room Screener Questionnaire}

Screeners are valuable in gaining complete data and helpful in directing care. Pro- 
viders use algorithms to identify differential diagnoses and the root cause of the symptoms. Getting an extensive history from IAVs can aid in understanding their relevant symptoms. According to The American Academy of Nursing (AAN), "Have you Ever Served in the Military?" campaign encourages providers to utilize screeners to address the needs of the veteran community. The AAN identifies exposure to open-air burn pits with "High risk for respiratory illnesses and wide variety of cancers, including leukemia". Therefore, focused screeners can contribute to proper assessments of symptoms associated with burn pit exposure and future care plans [20] A universal burn pit exposure screener does not exist at this time for civilian healthcare providers. However, a screener (Table 1) can be easily built into existing Electronic Health Record (EHR) to populate when a Veteran has been identified.

When IAVs with a history of burn pit exposures complain of respiratory issues, diagnostic studies can assist in proper diagnosis. Conducting serial PFTs and documenting spirometry changes in IAVs with recurrent respiratory issues can aid in understanding the trend of respiratory deterioration. Skin testing for allergies can assist in understanding allergic reactions from patients with burn pit exposures. Performing fractional exhaled nitric oxide examination can help in determining the severity of asthma issues. Conducting impulse oscillometry examinations can help in measuring small airway narrowing. If an IAV has had biopsies done, following up on the results in a timely manner is key to understanding the pathology of the disease. Providing resources and education on burn pit registries can help increase participation and completion. Diagnosing and documenting interventions can aid IAVs in accessing resources that can help them with financial hardship related to their medical conditions. There is a need for more research due to the gap of knowledge on health problems associated with burn pit exposure. However, waiting for years of research to be completed and then identifying and addressing this vulnerable population is not proactive healthcare. Thus, after initial treatment and stabilization of the patients' condition, the follow-up is key to the continuity of care and understanding the trend of health risks associated with burn pit exposure.

\section{Discussion and Conclusion}

Paul Rieckhoff of Iraq and Afghanistan Veterans of America calls burn pits "this generation's Agent Orange”. The US Supreme Court refused to hear an appeal by Iraq and Afghanistan veterans who claimed their illnesses were a direct result of their exposure to the toxic fumes associated with burn pits after a decade-long fight in the courts [21]. The Department of Veterans Affairs has no official stance on burn pits as it continues to study burn-pit-related claims. In recent years, burn pit exposure has received media coverage due to a growing concern with related health issues. The problem requires Congressional intervention, as demonstrated, as a first step, in the H.R 5671 Burn Pits Accountability Act. This bill was passed to track information with registries and aid in determining the

role of burn pits in veteran health issues. However, some of the veterans that 
seek treatment at the VA are not aware of these registries and/or are improperly assessed for possible health issues. Many veterans seek care outside the VA healthcare

Table 1. Burn pit screener (Adapted from burn pit 360, VA burn pit registry, and associated risk factors with burn pit exposure).

Have you ever served?

Where and for how long did you serve?

Were you deployed to the Middle East (Iraq and Afghanistan)?

Have you been exposed to burn pits? If so, did you wear a mask?

If you have been exposed to burn pits, how close and how long was your exposure?

Have you ever needed supplementary oxygen? If so, how often and for how long did you need it?

Have you ever had a pulmonary function test?

Have you ever had an impulse oscillometry?

Have you ever had a lung biopsy?

Have you ever experienced shortness of breath? If so, when did it occur (e.g., when exercising, when walking around the block, when walking from one room to another)?

Have you ever smoked? If so, when did you start smoking, and how frequently, how much, and for how long have you smoked? If you have quit smoking, when did you quit?

What type of environmental exposures have you had (e.g., dust storms, diesel fuel, depleted uranium, pesticides)?

Have you been diagnosed with any of the following?

- Arthritis/rheumatism

- Cancer

- Hypertension

- Brain injury (TBI)

- Fibromyalgia, lupus

- Migraine headaches

- Contact dermatitis

- COPD

- Asthma

- Allergies

- Vocal cord dysfunction

- Constrictive bronchitis

- Chronic fatigue syndrome

- Acid reflux/heartburn

- Blurred vision

- Eosinophilia esophagitis

- Chronic multisystem illness

- Chronic cough

- Memory loss

- Weight loss 
system, and their pertinent medical history is not well documented. Frontline providers and nurses must be well informed, recognize burn pit exposure, and take immediate action by directing the necessary resources toward the veteran's plan of care. Better education regarding health concerns related to burn pit exposure, an imbedded Electronic Health Record screener questionnaire, and a proactive civilian healthcare system that is invested in the health of our veteran community can help advocate and guide the care for veterans and voice the need for further research.

\section{Conflicts of Interest}

The authors declare no conflicts of interest regarding the publication of this paper.

\section{References}

[1] Goepner, E. (2018) War State, Trauma State: Why Afghanistan Remains Stuck in Conflict.

https://www.cato.org/publications/policy-analysis/war-state-trauma-state-why-afgh anistan-remains-stuck-conflict

[2] Institute of Medicine (2011) Long-Term Health Consequences of Exposure to Burn Pits in Iraq and Afghanistan. The National Academies Press, Washington, DC. http://nationalacademies.org/hmd/Reports/2011/Long-Term-Health-Consequences -of-Exposure-to-Burn-Pits-in-Iraq-and-Afghanistan.aspx

[3] Savitz, D.A., Styka, A.N. and Butler, D.A. (2017) Assessment of the Department of Veterans Affairs Airborne Hazards and Open Burn Pit Registry. The National Academies Press, Washington, DC. https://doi.org/10.17226/23677

[4] Iraq and Afghanistan Veterans of America (IAVA) (2019) Drive Support for Injuries from Burn Pits and Other Toxic Exposures.

https://iava.org/initiate-support-for-injuries-from-burn-pits-and-other-toxic-expos $\underline{\text { ures/ }}$

[5] Johnson, B.S., Boudiab, L.D., Freundl, M., Anthony, M., Gmerek, G.B. and Carter, J. (2013) Enhancing Veteran-Centered Care. American Journal of Nursing, 113, 24-39.

[6] Pugh, M.J., Jaramillo, C.A., Leung, K., Faverio, P., Fleming, N., Mortensen, E. and Morris, M.J. (2016) Increasing Prevalence of Chronic Lung Disease in Veterans of the Wars in Iraq and Afghanistan. Military Medicine, 181, 476-481.

https://doi.org/10.7205/MILMED-D-15-00035

[7] Szema, A., Mirsaidi, N., Patel, B., Viens, L., Forsyth, E., Li, J. and Burns, M. (2015) Proposed Iraq/Afghanistan War-Lung Injury (IAW-LI) Clinical Practice Recommendations: National Academy of Sciences' Institute of Medicine Burn Pits Workshop. American Journal of Men's Health, 11, 1653-1663.

https://doi.org/10.1177/1557988315619005

[8] Falvo, M.J., Helmer, D.A., Klein, J.C., Osinubi, O.Y., Ndirangu, D., Patrick-Deluca, L.A. and Sotolongo, A.M. (2016) Isolated Diffusing Capacity Reduction Is a Common Clinical Presentation in Deployed Iraq and Afghanistan Veterans with Deployment-Related Environmental Exposures. The Clinical Respiratory Journal, 12, 795-798. https://doi.org/10.1111/crj.12552 
[9] Krefft, S.D., Meehan, R. and Rose, C.S. (2015) Emerging Spectrum of Deployment-Related Respiratory Diseases. Current Opinion in Pulmonary Medicine, 21, 185-192. https://doi.org/10.1097/MCP.0000000000000143

[10] Liu, J., Lezama, N., Gasper, J., Kawata, J., Morley, S., Helmer, D. and Ciminera, P. (2016) Burn Pit Emissions Exposure and Respiratory and Cardiovascular Conditions among Airborne Hazards and Open Burn Pit Registry Participants. Journal of Occupational and Environmental Medicine, 58, e249-e255.

[11] Barth, S.K., Dursa, E.K., Bossarte, R. and Schneiderman, A. (2016) Lifetime Prevalence of Respiratory Diseases and Exposures among Veterans of Operation Enduring Freedom and Operation Iraqi Freedom Veterans. Journal of Occupational and Environmental Medicine, 58, 1175-1180.

[12] King, M.S., Eisenberg, R., Newman, J.H., Tolle, J.J., Harrell, F.E., Nian, H. and Miller, R.F. (2011) Constrictive Bronchiolitis in Soldiers Returning from Iraq and Afghanistan. New England Journal of Medicine, 365, 222-230. https://doi.org/10.1056/NEJMoa1101388

[13] Lentz, R.J., Fessel, J.P., Johnson, J.E., Maldonado, F., Miller, R.F. and Rickman, O.B. (2016) Transbronchial Cryobiopsy Can Diagnose Constrictive Bronchiolitis in Veterans of Recent Conflicts in the Middle East. American Journal of Respiratory and Critical Care Medicine, 193, 806-808. https://doi.org/10.1164/rccm.201509-1724LE

[14] Kingsbery, J. and Poles, J. (2018) Mo1054-Eosinophilic Esophagitis Following Exposure to Burn Pits during Military Deployment. Gastroenterology, 154, S-685. https://doi.org/10.1016/S0016-5085(18)32415-6

[15] Chiaramonte, P. (2018) Burn-Pit Exposure Likely Leads to Higher Cancer Mortality Rate among Army Vets: New study.

https://www.foxnews.com/us/burn-pit-exposure-likely-leads-to-higher-cancer-mort ality-rate-among-army-vets-new-study

[16] Wiedinmyer, C., Yokelson, R.J. and Gullett, B.K. (2014) Global Emissions of Trace Gases, Particulate Matter, and Hazardous Air Pollutants from Open Burning of Domestic Waste. Environmental Science \& Technology, 48, 9523-9530. https://doi.org/10.1021/es502250z

[17] (2018) As If You're Inhaling Your Death: The Health Risks of Burning Waste in Lebanon.

https://www.hrw.org/report/2017/12/01/if-youre-inhaling-your-death/health-risksburning-waste-lebanon

[18] Burn Pits 360 (n.d.) https://www.burnpits360.org/

[19] Abbasi, J. (2017) National Academies Report Cites Flaws in VA's Burn Pit Registry. JAMA: The Journal of the American Medical Association, 317, 1614. https://doi.org/10.1001/jama.2017.4509

[20] Intake Questions (n.d.) http://www.haveyoueverserved.com/intake-questions.html

[21] (2019) Supreme Court Rejects Appeal over Military Burn Pits. https://www.apnews.com/bd2443b13c844d60b8d37125ca3ab8bf 


\section{Abbreviations}

IAV

DOD

PTSD

TBI

VA

DLCO

$\mathrm{VCD}$

PFT

COPD

IED

EoE

CMI

$\mathrm{PAH}$

$\mathrm{AH} \& \mathrm{OBP}$

FOB

AAN
Iraq and Afghanistan veterans

Department of Defense

Post traumatic stress disorder

Traumatic brain injury

Veteran Affairs

Diffusing capacity for carbon monoxide

Vocal cord dysfunction

Pulmonary function test

Chronic obstructive pulmonary disease

Improvised explosive device

Eosinophilic esophagitis

Chronic multisystem illness

Polycyclic aromatic hydrocarbons

Airborne Hazard and Open Burn Pit

Forward operating base

American Academy of Nursing 